\title{
AC 2010-1206: A VIRTUAL ASSEMBLY SYSTEM ON AUTOMOBILE ENGINE FOR ASSEMBLY SKILLS TRAINING
}

Xiangchun Cheng, Zhejiang University of Technology

College of Education, Zhejiang University of Technology, Hangzhou, Zhejiang, 310014, China

\section{Rong Gu, WWW.ZJUT.EDU.CN}

College of Education, Zhejiang University of Technology, Hangzhou, Zhejiang, 310014, China

Min Chen, Zhejiang University of Technology

Yingzhen Weng, Zhejiang University of Technology 


\title{
A Virtual Assembly System on Automobile Engine for Assembly Skills Training
}

\begin{abstract}
Virtual Reality (VR) is wildly used in education and training, commonly associated with its immersive, highly visual, three dimension (3D) environments. This paper presents a virtual assembly system on automobile engine based on VR and simulation techniques. In order to improve the effects of skills training and make the system more scientific, the author introduced cognitive psychology into the design process. This system can be used as a support tool for skills training in automobile engine assembly, providing learners with virtual experience as a supplement to inadequate and insufficient real-world hands-on experience in highly visual 3D environment.
\end{abstract}

\section{Introduction}

Since the National Research Council issued a study that spotlighted the need for universities to graduate engineers with professional skills ${ }^{1}$ in 1985 , educators and researchers all over the world have made lots of efforts to promote development of engineering education.

As in China, evidence indicates that China has the largest number of engineering students, who are the reserve forces of future engineers; however, the quality of engineering education is quite low ${ }^{2}$. Come up with engineering undergraduates' lack of hands-on experience, engineering practice teaching and hands-on learning ${ }^{3}$ have increasingly become the focus of engineering education ${ }^{4}$. However, most of current training methods, paper-based manuals, video-based instructions and/or hands on master-apprentice training, can hardly solve the complicated engineering problem effectively.

Virtual Reality (VR) is a technology which allows a user to interact with computer-simulated environment. Recently, VR is wildly used in education and training, commonly associated with its immersive, highly visual, three dimension (3D) environments. As a new learning medium, VR can provide learners a hand-on experience with its interactive character, which can improve the learning performance effectively ${ }^{5}$. Practicing assembly tasks in the virtual environment helps facilitate training and aids in transferring that knowledge to real life. ${ }^{6}$ At the same time, VR can effectively resolve the time, space and security constraints problems brought about by actual equipments.

This paper presents a Virtual Assembly System $^{7-8}$ on Automobile Engine (AEVAS) based on VR and simulation techniques. This system aims to improve existing training methods by using a multidimensional, interactive virtual environment. The AEVAS is composed of the following four modules: Knowledge Room, Assembly Room, Expert Room and Checking Room. Knowledge Room provides 3D models and related details of the parts of the automobile engine so that the trainees can learn to recognize parts. It provides a necessary pre-knowledge for other three modules. Assembly Room mainly provides interactive 3D demonstration animations so that the trainees can remember assembly sequences, orients and positions; another function of this 
module is the trainees can get experience of assembly by using 3D joystick to move and fix parts in the virtual environment. Expert Room is served as assistance that can give helps information and check for user errors in the training progress; also it can record the progress of the manipulations so that the trainees can watch the record after training. Checking Room is a module, which is quite a new attempt, for promotion by taking away a part of the engine randomly and let the user to judge where to re-put it on correctly.

This paper mainly includes five parts: System Requirement, System Framework Design, System Implementation, Education Application, Discussion and Conclusion. System Requirement part discusses the system goals and the functions the system should include. In System Framework Design part, we propose the main modules of the system and discuss rationale behind design decisions. Then the main implementation of the system is described in System Implementation part. After that, we describe testing and results. Final is the discussion and conclusion.

\section{System Requirement}

The main goal of the AEVAS is to make the assembly training process of automobile engine more convenient, economic and effective by using a 3D joystick to interact with multi-modal virtual environment.

By analyzing the requirement of real automobile engine assembly skill acquisition and human cognitive process, here are the functions our system should include:

- Knowledge: 3D models of every part of the automobile engine and related information for trainees to recognize the parts.

- Interaction: all the 3D models can be rotated and zoomed displayed conveniently connecting with related information when chosen.

- Reliability: the process of virtual assembly should have same rules as real assembly, such as assembly positions and sequences, and the parts models can be moved in three dimensions.

- Help: help functions during the process of assembly: warning tones, tooltips and color changing for attention.

- Feedback: the system can provide trainees with feedbacks of their evaluation after training.

- Easiness: the manipulation of assembly should be performed easily.

\section{System Framework Design}

Four-component Instruction Design Model (4C/ID) ${ }^{9}$ is a theory model for design of training programs for complex skills. According to its four components: learning tasks, supportive information, just-in-time (JIT) information and part-task practice, by analyzing the skills of automobile engine assembly together with documents investigation and interview to the master about the engine assembly, we designed the framework of the AEVAS as shown in Fig.1. 


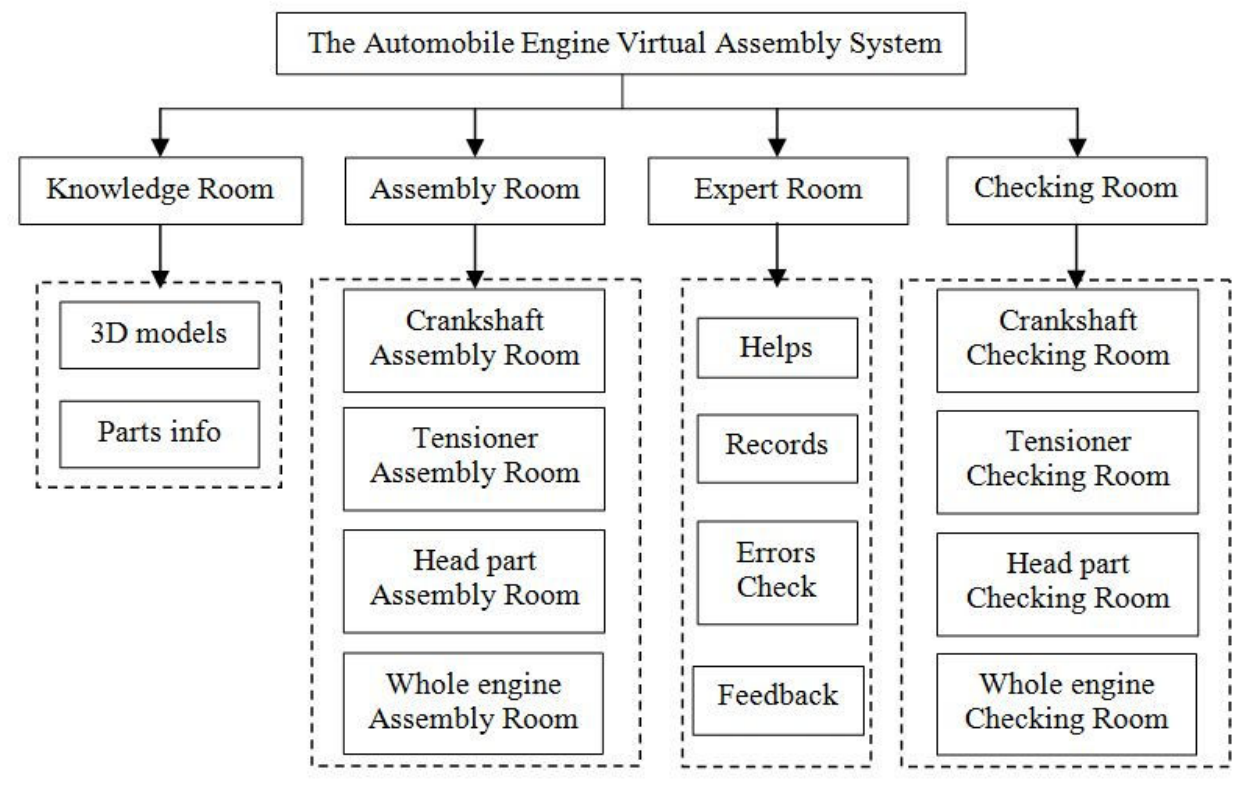

Figure 1. The Framework of Automobile Engine Virtual Assembly System

The AEVAS is composed of the following four modules: Knowledge Room, Assembly Room, Expert Room and Checking Room.

- Knowledge Room: Knowledge Room is the first part after the trainees login the system. Trainees can get the related texts, photos and videos info on the parts. By learning here, the trainees can recognize the $3 \mathrm{D}$ parts and get the related pre-knowledge.

- Assembly Room: according to part-task practice, one of the components of 4C/ID Model, we set the Assembly Room to four parts: Crankshaft Assembly Room, Head Assembly Room, Tensioner Assembly Room and the Whole Engine Assembly Room. Each room has a parts exhibition function, provides interactive 3D animations of the process of the assembly to help users know the rough sequences and final manipulate interaction process of assembly.

- Expert Room: this is an assistance part implanted in the Assembly Room. It mainly contains following function: manipulation introductions which can be hidden, tooltips and warning tones when a user input is erroneous, color changing when the part is in the right position and rotation, records of the trainees' manipulation process and some other simple feedbacks.

- Checking Room: this is a new attempt to add this function, here the system would take a part away and the trainees should judge where and in which sequences to re-put it on. 


\section{System Implementation}

The main software we used in exploitation is Pro/Engine (Pro/E) and Quest 3D (Q3D). Pro/E is a parametric, integrated 3D CAD/CAM/CAE solution created by Parametric Technology Corporation (PTC) which is powerful for mechanical engineering, design and manufacturing. Q3D is the perfect solution for architecture visualization, product visualization, digital entertainment, computer aided training and high-end VR applications.

- Modeling and Information Database: according to the real engine parts and the demand of the learners, use 3D modeling software Pro-E to model the parts of the engine and set the relative knowledge to build up info-database with text, photos and videos. Models are shown in Fig.2.
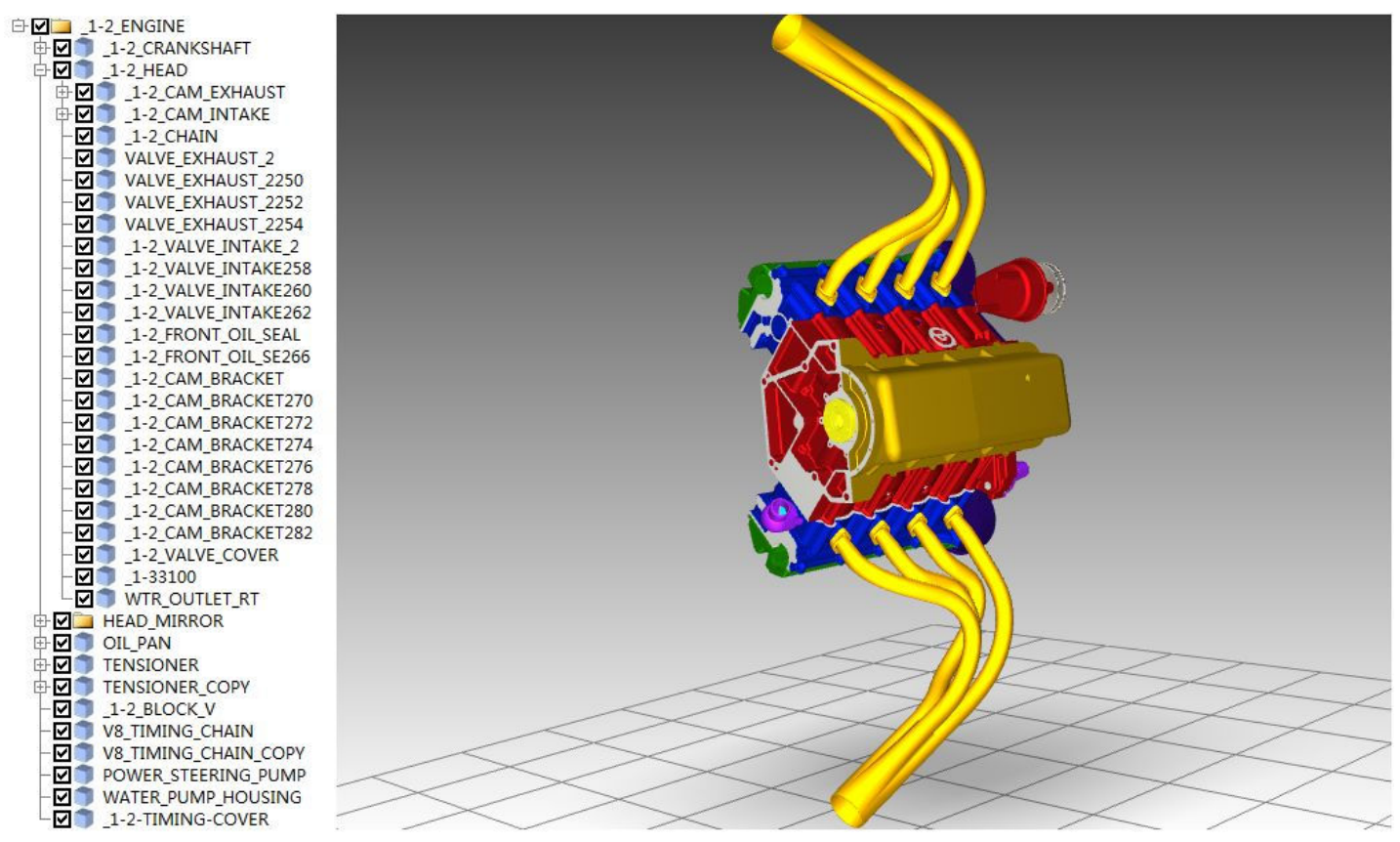

Figure 2. The 3D Parts Models of the Automobile Engines 
- Pre-treatment: import the parts of the engine into 3D interacting software Quest 3D to pick up the relative restrictions of virtual assembly and to deal with sequences by a value channel.

- Interaction: set the X/Y/Z position/rotation value channel to connect with a 3D joystick input channel in Quest to let the movement/rotation, and set viewpoint of assembly can be zoomed in/out and rotated by using inspection camera together with a basic one.

- Help Functions: we set some restrains by calculating the number value of the parts to give warning tones with tooltips by sound and text-out channel when the trainees select not in right sequences.

The Fig. 3 is a small module for trainees to recognize the parts before related assembly training. The Fig. 4 is the Whole Engine Assembly Room. The Fig.5 is the Crankshaft Assembly Room and Fig.6 is the helps during the process of assembly.

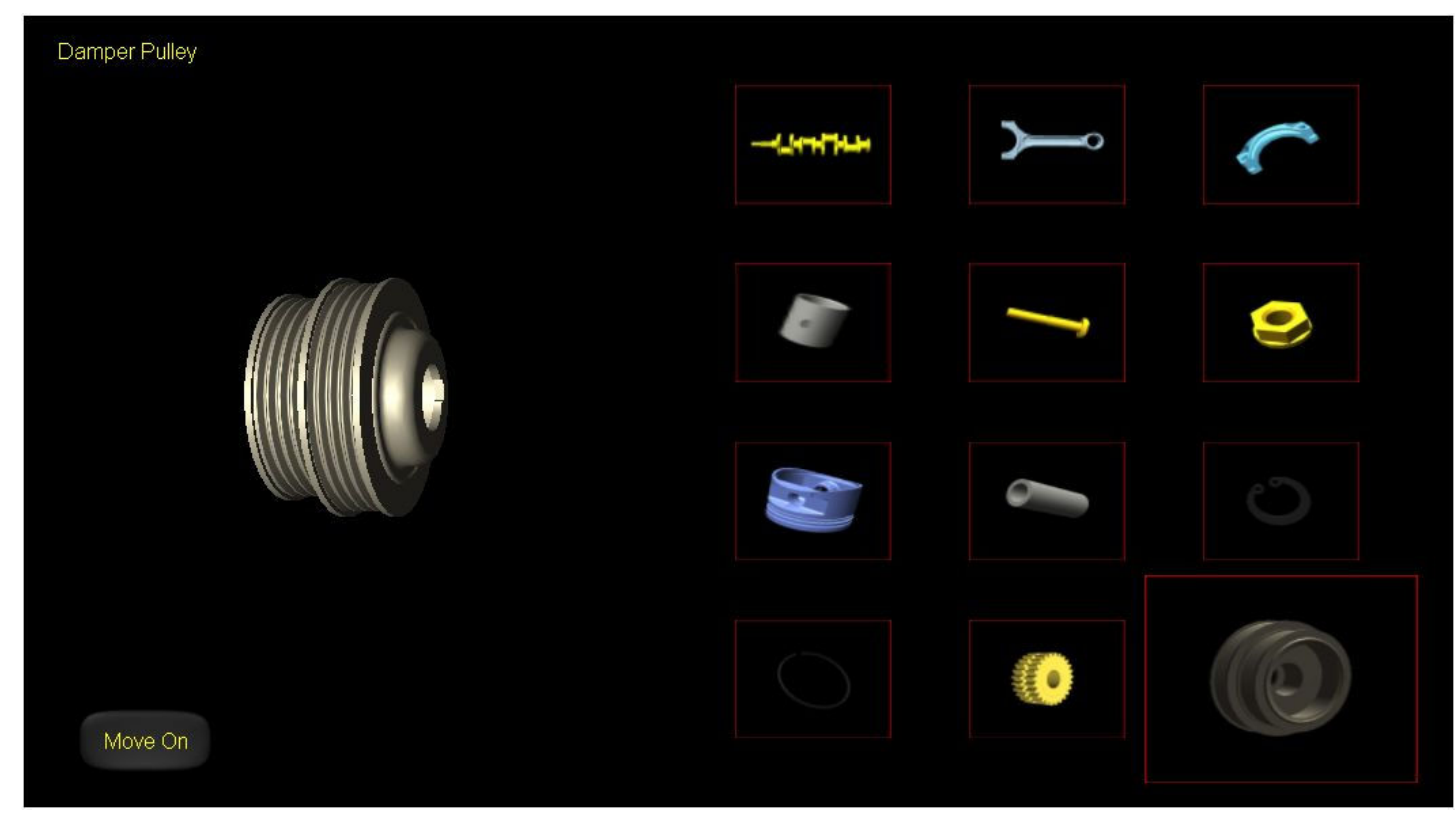

Figure 3. Knowledge Room 


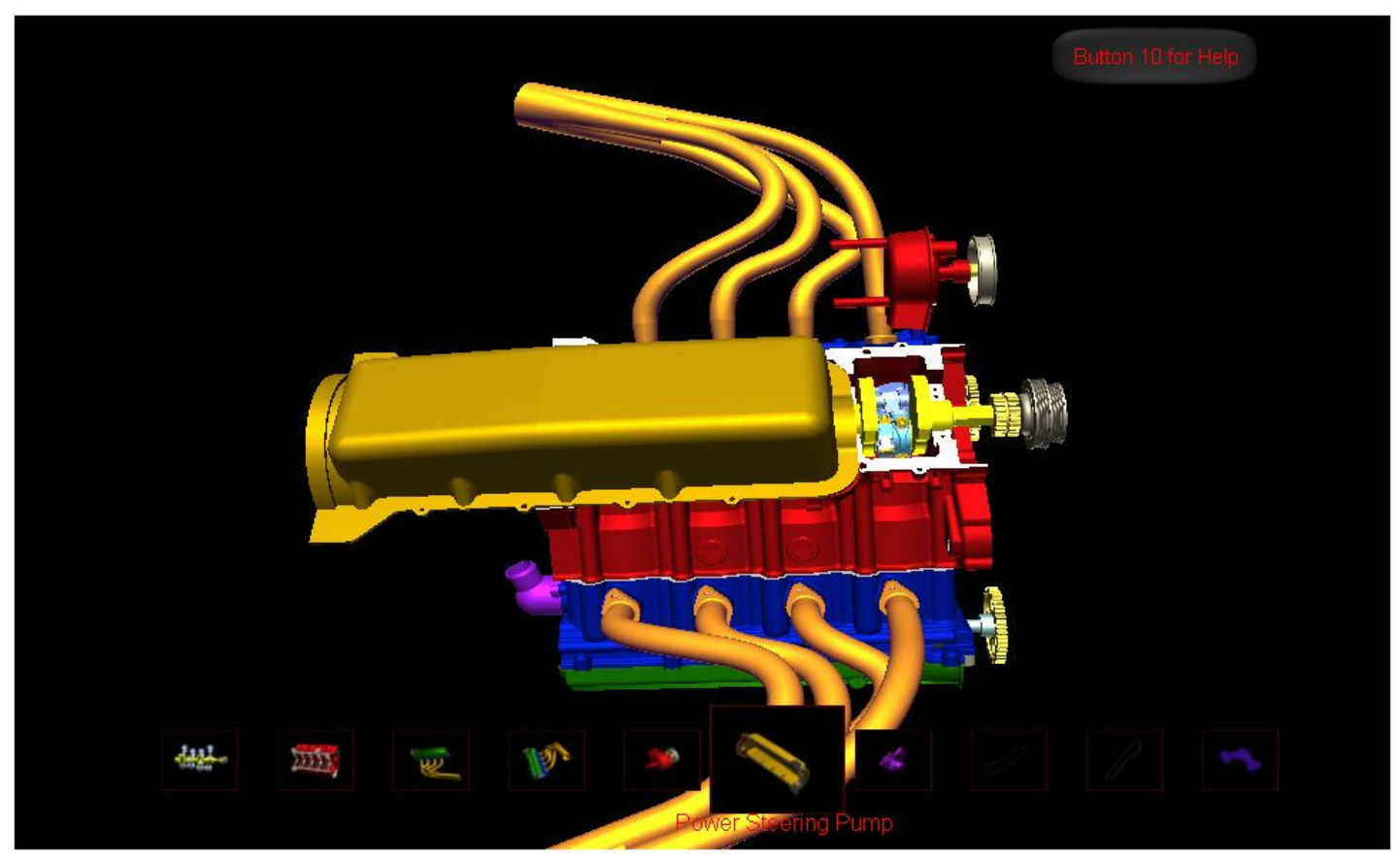

Figure 4. Whole Engine Assembly Room

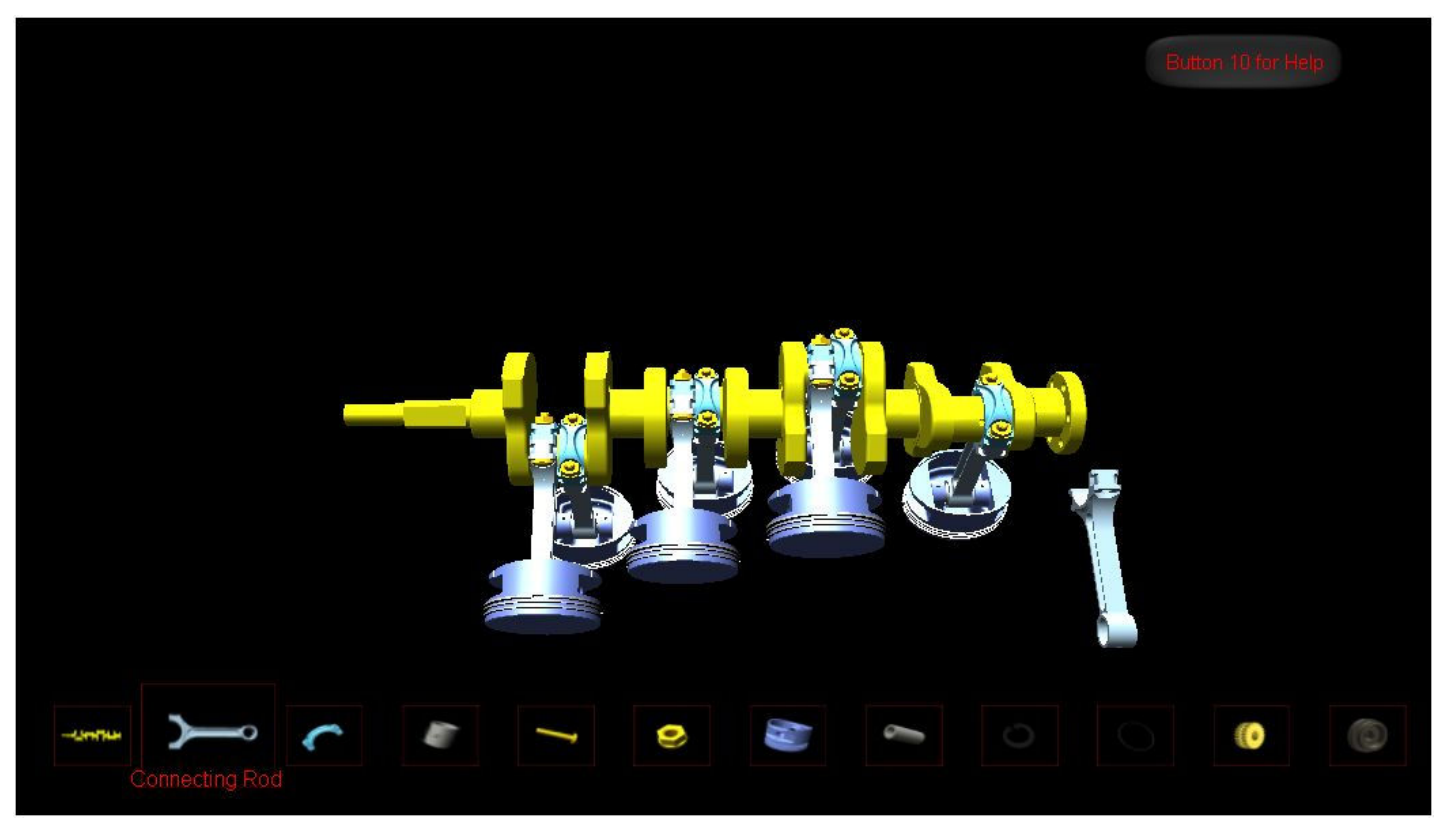

Figure 5. Crankshaft Assembly Room

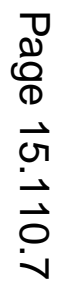




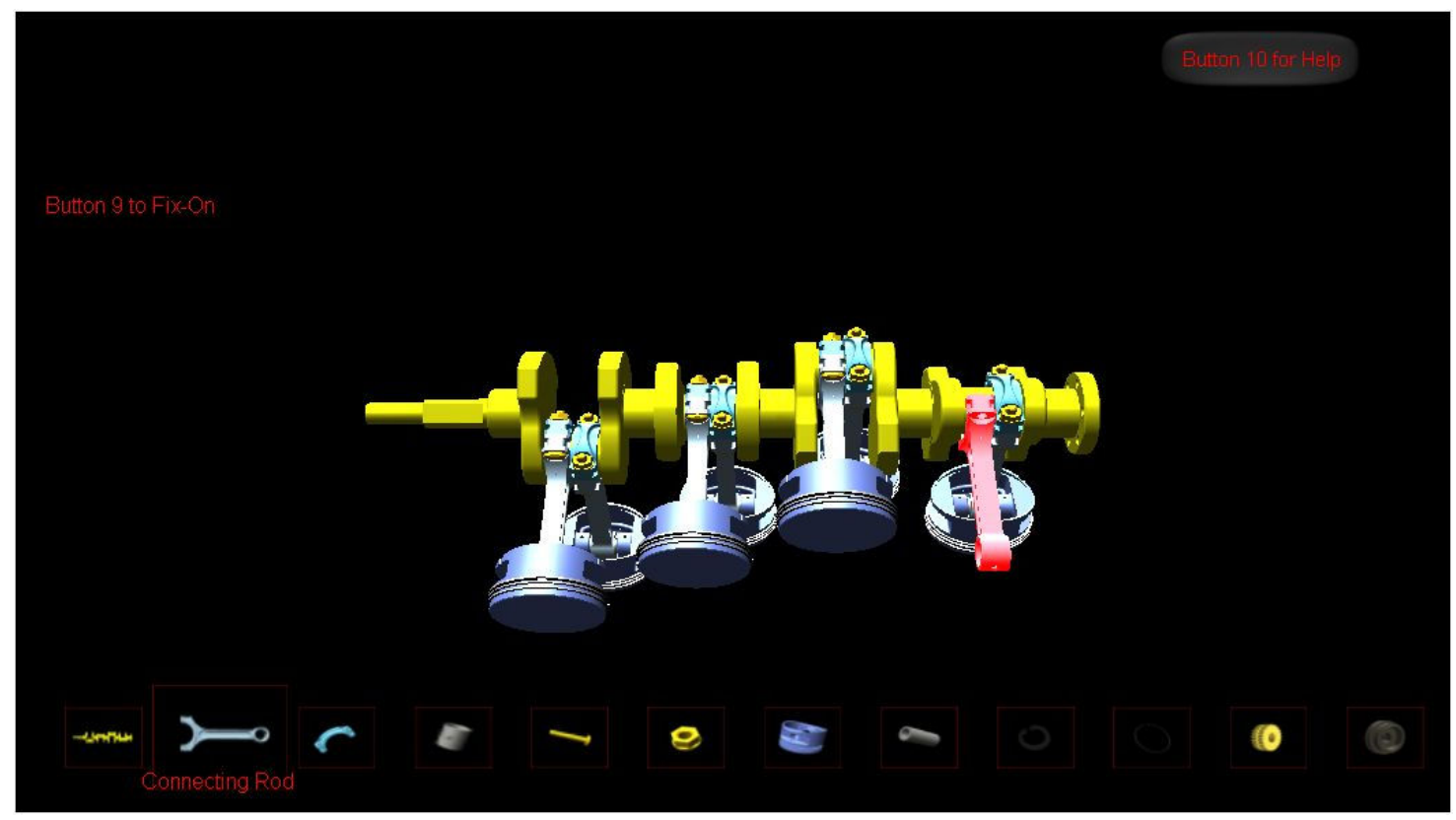

Figure 6. Tooltips and Color Changing

\section{Testing and Results}

We conducted a detailed user study involving 30 subjects for 2 weeks to get performance of this system. The subjects are composed of twenty employees selected from three different companies of automobile engine and ten undergraduate students of engineering schools. Fig.7 shows the main results.

Table 1. Main Assess Results

\begin{tabular}{|l|c|c|c|c|c|}
\hline \multirow{2}{*}{ Assess matters } & \multicolumn{5}{|c|}{ Assess results(sums of trainees) } \\
\cline { 2 - 6 } & $\begin{array}{l}\text { Very } \\
\text { Satisfied }\end{array}$ & Satisfied & Common & $\begin{array}{l}\text { Not } \\
\text { good }\end{array}$ & Very Bad \\
\hline Content Organization & 9 & 18 & 3 & & \\
\hline Training Strategies & 12 & 15 & 3 & & \\
\hline Training targets & 3 & 16 & 9 & 2 & \\
\hline The overall effect & 6 & 21 & 3 & & \\
\hline
\end{tabular}




\section{Discussion and Conclusion}

In this study, we investigated the framework and the training process of the AEVAS in virtual learning environments for assembly skills training. Virtual learning environments offer the possibility to recreate the real world as it is, providing virtual experience that can help people in understanding concepts as well as learning the process of performing special tasks, where the task can be repeated as often as required and in a safe environment ${ }^{10}$.

The AEVAS is a virtual assembly system on automobile engine based on VR and simulation techniques. This system can be used as a support tool for skills training in automobile engine assembly, providing learners with virtual experience as a supplement to inadequate and insufficient real-world hands-on experience in highly visual 3D environment.

\section{Acknowledgement}

The work is supported by Innovation Experiment Program for university students of China.

\section{Bibliography}

1. Engineering Education and Practice in the United States: Foundations of our Techno-Economic Future. (1985). Washington, D.C., National Research Council.

2. L.Y.Cheng. (2008). Reform Engineering Education and Build up China's Strength in Engineering Education. Sep, 2008. Education and Modernization, China.

3. Engineering in the K-12 Classroom an Analysis of Current Practices \& Guidelines for the Future. (2004). the American Society for Engineering Education. Washington, D.C.

4. Z.P.Ye and P.Hua Jin. (2007). A Review of Studies on Practice Teaching of Engineering Education in China. Apr, 2007. Research in Higher Education of Engineering, China.

5. Paul Kirschner and Peter Gerjets. (2006). Instructional Design for Effective and Enjoyable Computer-Supported Learning. Jan, 2006. Computers in Human Behavior.

6. John E. Brough, Maxim Schwartz, Satyandra K. Gupta , Davinder K. Anand, Robert Kavetsky and Ralph Pettersen. (2007).Towards the development of a virtual environment-based training system for mechanical assembly operations. Mar, 2007. Springer.

7. P. Long, S. Liu, Y. Wu and the FDS Team. (2007). Design and Testing of the Fusion Virtual Assembly Simulation. Oct, 2007. Fusion Engineering and Design, China.

8. Gabriel Zachmann and Alexander Rettig. (2001). Natural and Robust Interaction in Virtual Assembly Simulation. Aug, 2001. Eighth ISPE International Conference.

9. Jeroen J. G. van Merriënboer, Richard E. Clark and Marcel B. M. de Croock. (2006). Blueprints for complex learning: The 4C/ID-mode. Sep, 2006. Educational Technology Research and Development.

10. Luca Chittaro, Roberto Ranon. (2007). Web3D technologies in learning, education and training: Motivations, issues, opportunities. 2007. Computers \& Education. 